\title{
GRB PROMPT OPTICAL/UV EMISSION AND DUST SUBLIMATION
}

\author{
XIAO-HONG CUI*, ZHUO LI ${ }^{\dagger}, \ddagger$ and LI-PING XIN* \\ *National Astronomical Observatories, Chinese Academy of Sciences \\ Beijing 100012, China \\ ${ }^{\dagger}$ Department of Astronomy, Peking University \\ Beijing 100871, China \\ ${ }^{\ddagger}$ Kavli Institute for Astronomy and Astrophysics, Peking University \\ Beijing 100871, China
}

\begin{abstract}
Observations imply that long $\gamma$-ray bursts (GRBs) are originated from explosions of massive stars, therefore they may occur in the molecular clouds where their progenitors were born. We show here that the prompt optical-UV emission from GRBs may be delayed due to the dust extinction, which can well explain the observed optical delayed onset and fast rise in GRB 080319B. The density and the size of the molecular cloud around GRB 080319B are roughly constrained to be $\sim 10^{3} \mathrm{~cm}^{-3}$ and $\sim 8 \mathrm{pc}$, respectively. We also investigate the other GRBs with prompt optical-UV data, and find similar values of the densities and sizes of the local molecular clouds. The future observations of prompt optical-UV emission from GRBs in subsecond timescale, e.g., by UFFO-Pathfinder and SVOM-GWAC, will provide more evidence and probes of the local GRB environments.
\end{abstract}

\section{Introduction}

The properties of $\gamma$-ray burst (GRB) circumburst and host-galaxy environment are important for the studies of GRB progenitors and the fundamental conditions required within a galaxy to form a GRB. The multi-wavelength observations about the emission from GRBs and that from their host galaxies would provide a unique tool to understand the nature of GRBs and the properties of interstellar medium (ISM) around the bursts.

In this work, we show that if a GRB is located in a molecular cloud, its prompt optical-UV emission may be absorbed by the dust in the molecular cloud, and only emerges after the dusts on the line of sigh are all destroyed. This can well explain the observed delayed onset of the prompt optical-UV emission in GRB 080319B, and the density and the size of the molecular cloud around this burst can be roughly constrained. For other bursts with prompt optical observations, the properties of local environment can also be constrained. We find similar properties of the clouds, with density and size being $n_{\mathrm{H}} \sim 10^{3}-10^{4} \mathrm{~cm}^{-3}$ and $\Delta R \sim 6 \mathrm{pc}$. 


\section{The Dust Sublimation Model and Applications}

Consider a GRB that is located in a molecular cloud. The prompt optical-UV emission from this GRB may be absorbed by the dust in the cloud, but in the same time the dusts may also be destroyed by the emission. If the optical-UV emission is strong and lasts long enough, it may emerge from the cloud after the dusts on the way are all destroyed. The dust destruction by the optical-UV radiation has been discussed by Waxman \& Draine (2000). Here we will follow their model in the radiation-dust interaction, and focus on the back effect of the dust on the optical-UV emission, i.e., how the dusts in a cloud of finite size affects the apparent light curve of the prompt optical-UV emission. On the other hand, from the observed light curve profile of the prompt optical-UV emission, we can also give some constraints on the properties of the molecular cloud. The temperature $T$ of the grain at distance $r$ from the source is governed by

$$
\frac{L_{1-7.5}}{4 \pi r^{2}} Q_{\mathrm{UV}} \pi a^{2}=\langle Q\rangle_{T} 4 \pi a^{2} \sigma T^{4}-4 \pi a^{2} \frac{d a}{d t} \frac{\rho}{m} B,
$$

where $m$ is the mean atomic mass, $B$ is the chemical binding energy per atom, $Q_{\mathrm{UV}}$ is the absorption efficiency factor averaged over the $1-7.5 \mathrm{eV}$ spectrum of the source emission, and $\langle Q\rangle_{T}$ is the usual Planck-averaged absorption efficiency.

Define $f(r)$ the fraction of the flash energy that is absorbed by dust interior to radius $r$. If $t_{\text {surv }}<(1-f) \Delta t$, the grains are destructed and $f(r)$ satisfies

$$
\frac{d f}{d r}=Q_{\mathrm{UV}} n_{d} \pi a^{2} \frac{t_{\mathrm{surv}}}{\Delta t} .
$$

The relation between the radius of dust destruction front $R_{f}$ and observer time $t_{\text {obs }}$ can be given by $t_{\text {obs }}=f\left(R_{f}\right) \Delta t(1+z)$, with $z$ the redshift of the GRB source. A (maximum) dust destruction radius $R_{d}$ is determined by the condition $t_{\text {surv }}\left[T\left(R_{d}\right)\right]=\left[1-f\left(R_{d}\right)\right] \Delta t$. If $R_{f}<\Delta R$, the optical depth due to dust extinction is $\tau=Q_{\mathrm{UV}} n_{d} \pi a^{2}\left(\Delta R-R_{f}\right)$, and the attenuated luminosity observed outside is

$$
L_{\text {obs }}\left(t_{\text {obs }}\right)=L_{1-7.5} \exp \left\{-\tau\left[R_{f}\left(t_{\text {obs }}\right)\right]\right\} .
$$

So far the so-called "naked-eye" GRB 080319B is the only one that happens to occur in the field of view of an optical telescope, without the trigger by high-energy detector, thus it is by luck monitored in optical band from before the beginning of the GRB. The broadband observations of it has been presented by Racusin et al. (2008) and Beskin et al. (2010).

In order to decouple $n_{\mathrm{H}}$ and $\Delta R$, we need to further consider the temporal profile of the observed optical-UV emission. For different values of $n_{\mathrm{H}}$ and $t_{b}$, we have calculated the optical-UV light curve using equation (3).Note, as Beskin et al. (2010) found that the optical emission is $2 \mathrm{~s}$ delayed relative to the $\gamma$-ray emission in the plateau phase, we also assume a time delay of $2 \mathrm{~s}$ for the intrinsic onset of opticalUV emission compared to the $\gamma$-ray one. We see that compared with the observed optical data of GRB $080319 \mathrm{~B}$, the case with $n_{\mathrm{H}}=10^{3} \mathrm{~cm}^{-3}$ and $t_{b}=16 \mathrm{~s}$ fits the 
Table 1. The observational results of GRBs in Sample I and the constraints of their local molecular clouds.

\begin{tabular}{cccccccccccc}
\hline GRB & $z$ & $\begin{array}{c}T_{90} \\
(\mathrm{~s})\end{array}$ & $\begin{array}{c}t_{\mathrm{op}} \\
(\mathrm{s})\end{array}$ & $\kappa^{*}$ & $\begin{array}{c}L_{1-7.5} \\
\left(10^{48} \mathrm{erg} \mathrm{s}^{-1}\right)\end{array}$ & $\begin{array}{c}R_{\mathrm{d}} \\
(\mathrm{pc})\end{array}$ & $\begin{array}{c}\Delta R \\
(\mathrm{pc})\end{array}$ & $\begin{array}{c}n_{\mathrm{H}} \\
\left(10^{3} \mathrm{~cm}^{-3}\right)\end{array}$ & $\begin{array}{c}\Delta t_{\mathrm{obs}} \\
(\mathrm{s})\end{array}$ & $\begin{array}{c}t_{b} \\
(\mathrm{~s})\end{array}$ & ref \\
\hline $050820 \mathrm{~A}$ & 2.6 & $750^{* *}$ & 84 & $3.10(\mathrm{R})$ & 0.6 & 3.35 & 3.23 & 9 & 646 & 305 & 1 \\
060418 & 1.49 & 103.1 & 40 & $4.07(\mathrm{H})$ & 2.4 & 6.91 & 6.87 & 4 & 140 & 107 & 2 \\
$060607 \mathrm{~A}$ & 3.082 & 102 & 73 & $4.07(\mathrm{H})$ & 2.3 & 5.90 & 5.87 & 5 & 200 & 150 & 2 \\
$080319 \mathrm{~B}$ & 0.937 & 57 & $8.9^{\dagger}$ & $6.17(\mathrm{~V})$ & 260 & 7.78 & 7.67 & 1 & 50 & 16 & 3,4 \\
080810 & 3.35 & 106 & 38 & $3.10(\mathrm{~W})$ & 7.1 & 11.7 & 11.4 & 3 & 150 & 67 & 5 \\
$100906 \mathrm{~A}$ & 1.727 & 114.4 & 48.5 & $3.10(\mathrm{~W})$ & 1.2 & 4.60 & 4.48 & 15 & 190 & 83 & 6 \\
\hline
\end{tabular}

*In the bracket is the passband of filter. Letters "V", "H" and "W" denote V, H and white bands, respectively.

** From the work of Vestrand et al. (2006), rather than Swift data.

${ }^{\dagger}$ The time corresponds to the first optical data by TORTORA. In fact, the optical observations start before the trigger of this GRB.

References: (1) Vestrand et al. 2006; (2) Molinari et al. 2007; (3) Racusin et al. 2008; (4) Beskin et al. 2010; (5) Page et al. 2009; (6) Gorbovskoy et al. 2011.

light curve profile better. Therefore, it can be concluded that the cloud that hosts GRB $080319 \mathrm{~B}$ has a density of $n_{\mathrm{H}} \approx 10^{3} \mathrm{~cm}^{-3}$ and a size of $R \sim \Delta R \approx 8$ pc. Besides GRB 080319B, there are quite a few other GRBs with prompt optical detections during the $\gamma$-ray bursting phase. They are all detected by rapid slew of optical telescopes to the GRB location after trigger by $\gamma$-ray detectors. So usually there is a gap between the trigger time and the start time of optical observation. Nevertheless, we can still try to make some constraints on the local GRB environments based on the simple radiation-dust interaction model.

All the GRBs detected after December of 2004 and with optical detections during the prompt $\gamma$-ray emission are collected and analyzed with the simple radiation-dust interaction model here. Given $L_{1-7.5}$ and $\Delta t$, the maximum dust destruction radius $R_{d}$ can be determined to be as function as density $n_{\mathrm{H}}$. Furthermore, the time $t_{b}$ that the optical flux rise to the top value can be estimated from the observed optical light curve. Once given $f(r=\Delta R)=t_{b} / \Delta t_{\mathrm{obs}}$ and combined with the condition of $R_{d}=\Delta R$, one obtains a minimum $n_{\mathrm{H}}$ value, $n_{\mathrm{H}}>n_{\mathrm{H}, 0}$, otherwise, the destruction front cannot reach the edge of the cloud and no optical-UV emission escapes from the cloud. Finally, we apply eq. (3) to fit the rising part of the optical light curve by taking $n_{\mathrm{H}}$ (in the range of $n_{\mathrm{H}}>n_{\mathrm{H}, 0}$ ) and $t_{b}$ as free parameters. The best fit gives us the resulted values of $n_{\mathrm{H}}$ and $\Delta R$.

The resulted values of $n_{\mathrm{H}}$ and $\Delta R$ is also listed in Table 1 . We find that the density of the surrounding molecular clouds are in the range of $10^{3}-10^{4} \mathrm{~cm}^{-3}$, while the size, as implicated by $\Delta R$, is in the order of $\sim 10$ pc. However, due to small number of GRBs with optical rising part detected in prompt emission, it is impossible to give the statistic discussion of the properties of local molecular clouds. Furthermore, the observed data points in the optical rising part are usually sparse for individual GRB, which may induce large errors in light curve fitting. The future 
precise observations are needed to test the model and constrain the properties of local environment more precisely.

\section{Conclusions}

Long GRBs are believed to be the explosions of massive stars, therefore the GRBs may occur in the molecular clouds where their progenitors were born. We show in this work that the prompt optical-UV emission from GRBs, if originally emitted simultaneously with $\gamma$-ray emission, may appear with relative time delay in observations, due to the dust extinction. This can well explain the optical delayed onset observed in GRB 080319B, and the number density and the size of the molecular cloud are roughly constrained to be $n_{\mathrm{H}} \sim 10^{3} \mathrm{~cm}^{-3}$ and $\Delta R \sim 8 \mathrm{pc}$, respectively. We also investigate the other GRBs with good optical-UV data, and find the densities and sizes of the molecular clouds in the range of $n_{\mathrm{H}} \sim 10^{3}-10^{4} \mathrm{~cm}^{-3}$ and $\Delta R \sim 10$ pc.

This work was supported by National Basic Research Program of China-973 Program 2009CB824800, China Postdoctoral Science Foundation funded project (No. 20110490590) and the Foundation for the Authors of National Excellent Doctoral Dissertations of China.

\section{References}

Beskin, G., Karpov, S., Bondar, S., et al. 2010, ApJ, 719, L10

Gorbovskoy, E. S., Lipunova, G. V., et al. 2011, preprint in MNRAS, arXiv:1111.3625

Molinari, E., Vergani, S. D., Malesani, D. et al., 2007, A\&A, 469, 13

Page, K. L., Willingale1, R., Bissaldi, E., et al., 2009, MNRAS, 400, 134

Racusin, J. L., Karpov, S. V., Sokolowski, M. et al., 2008, Nature, 455, 183

Waxman, E. \& Draine, B. T. 2000, ApJ, 537, 796 